\title{
Hybrid surface plasmon polaritons in graphene coupled anisotropic van der Waals material waveguides
}

\author{
Hodjat Hajian ${ }^{1, *}\left(\mathbb{D}\right.$, Ivan D Rukhlenko ${ }^{2,3}\left(\mathbb{D}\right.$, George W Hanson ${ }^{4}\left(\mathbb{D}\right.$ and Ekmel Ozbay ${ }^{1,5,6, *}$ (i) \\ ${ }^{1}$ NANOTAM-Nanotechnology Research Center, Bilkent University, Ankara 06800, Turkey \\ ${ }^{2}$ The University of Sydney, Institute of Photonics and Optical Science (IPOS), School of Physics, \\ Camperdown 2006 NSW, Australia \\ ${ }^{3}$ Information Optical Technologies Centre, ITMO University, Saint Petersburg 197101, Russia \\ ${ }^{4}$ Department of Electrical Engineering, University of Wisconsin, 3200 North Cramer Street, Milwaukee, \\ WI 53211, United States of America \\ ${ }^{5}$ Department of Electrical and Electronics Engineering, Bilkent University, Ankara 06800, Turkey \\ ${ }^{6}$ Department of Physics and UNAM-Institute of Materials Science and Nanotechnology, Bilkent \\ University, Ankara 06800, Turkey \\ E-mail: hodjat.hajian@bilkent.edu.tr and ozbay@bilkent.edu.tr
}

Received 4 June 2021, revised 28 July 2021

Accepted for publication 9 August 2021

Published 23 August 2021

\begin{abstract}
Polaritons in anisotropic van der Waals materials (AvdWMs), with either hyperbolic or elliptical topologies, have garnered significant attention due to their ability of field confinement and many useful applications in in-plane polariton nanophotonics, including directional guiding, canalization, and hyperlensing. Here, we obtain the dispersion relation of hybrid surface plasmon polaritons (SPPs) supported by a parallel-plate waveguide composed of an AvdWM, as an example tungsten ditelluride, that is coupled with a graphene layer. Through analytical calculations and numerical simulations, we first investigate the impact of losses on the modal characteristics of SPPs supported by the AvdWM. We then show that the coupling of the anisotropic layer to a graphene sheet in a parallel-plate waveguide heterostructure allows one to control the in-plane propagation and dispersion topology of the hybrid SPPs by changing the spacer thickness and the graphene chemical potential. Moreover, it is found that owing to the different coupling regimes, this anisotropic-isotropic SPPs hybridization can enhance the propagation length and spatial localization of the guided modes. We believe this approach can lead to the realization of vdW heterostructures with improved functionalities for in-plane and out-of-plane infrared nanophotonics.
\end{abstract}

Supplementary material for this article is available online

Keywords: anisotropic, van der Waals materials, graphene, surface plasmon polaritons, dispersion topology

(Some figures may appear in colour only in the online journal)

\footnotetext{
* Authors to whom any correspondence should be addressed.
} 


\section{Introduction}

Polaritons with in-plane hyperbolic or elliptic momentum topology (i.e. with hyperbolic or elliptic isofrequency contours (IFCs) in the momentum space) have been employed for studying a wide variety of exotic optical phenomena. Some examples include topological transitions [1], directional, unidirectional, and asymmetric guiding [1-11], canalization $[11,12]$, the enhancement of local density of states and planar hyperlensing [13], negative refraction [4], and superCoulombic atom-atom interactions [14]. Polaritons exhibiting in-plane anisotropy were first realized in uniaxial metasurfaces (characterized by unequal components of the in-plane effective permittivity tensor) made of plasmonic materials, such as Ag [2-4] and graphene [1]. The subsequent employment of hexagonal boron nitride (hBN) supporting phonon polaritons with an out-of-plane hyperbolic response [15, 16] showed that uniaxial hBN-based metasurfaces can also guide polaritonic rays with the in-plane hyperbolic topology $[17,18]$.

There are two factors limiting the capability of uniaxial metasurfaces: fabrication inaccuracies due to the imperfections of the lithography processes and the limitations in the momentum space imposed on the hyperbolic regime by the finiteness of the meta-atom size. To overcome these limitations, plasmonic van der Waals (vdW) materials with an inplane anisotropic response, most notably black phosphorous, have been theoretically suggested to naturally sustain hyperbolic polaritons in the infrared spectral range [6-13, 19-22]. It has been later shown experimentally that the surface of $\alpha$-phase molybdenum trioxide $\left(\alpha-\mathrm{MoO}_{3}\right)$, which is a wellknown member of the vdW material family, can support the propagation of anisotropic phonon polaritons in the midinfrared range [23-27]. Infrared nanoimaging and nanospectroscopy of $\alpha-\mathrm{MoO}_{3}$ disks demonstrated that phonon polaritons with elliptic and hyperbolic in-plane dispersions can exist in the Reststrahlen band of $\alpha-\mathrm{MoO}_{3}$. It was also found that the wavelengths of the anisotropic phonon polaritons of $\alpha-\mathrm{MoO}_{3}$, while being up to 60 times smaller than the respective photon wavelengths, are comparable to the wavelengths of graphene plasmon polaritons [28-30] and boron nitride phonon polaritons [16]. The in-plane polaritonic anisotropy is further enhanced in vdW heterostructures such as hBN/black phosphorus (BP) [31], where the BP anisotropic response hybridized with the $\mathrm{hBN}$ phonons and where one can observe topological transitions of the hybrid modes within the second Reststrahlen band of hBN [11]. This approach offers an additional degree of freedom for controlling the propagation of in-plane anisotropic polaritons in lithography-free planar vdW heterostructures.

In contrast to uniaxial metasurfaces, natural hyperbolic materials possess stronger electromagnetic confinement and a more divergent photonic density of states [19, 20]. More importantly, their hyperbolic regime can be extended to the mid-infrared and terahertz frequencies, which correspond to molecular vibrations and thermal radiation [32]. While this feature is highly beneficial for chemical sensing and heat management, the fixed position of the $\alpha-\mathrm{MoO}_{3}$ Reststrahlen band severely limits its technological applications. One way of overcoming this issue is to chemically switch the phononic response of $\alpha-\mathrm{MoO}_{3}$ by engineering the $\alpha-\mathrm{MoO}_{3}$ crystal through intercalation with metallic $\mathrm{Sn}, \mathrm{Co}$, and $\mathrm{Cu}$ [33] or hydrogen [34]. This method has been recently used to demonstrate that the intercalation of $\mathrm{Na}$ atoms in $\alpha-\mathrm{V}_{2} \mathrm{O}_{5}$ produces a large spectral shift of the Reststrahlen bands of this vdW semiconductor while opening up a route to engineering the response of its phonon polaritons [35]. Another way of realizing spectral tunability relies on the recent observation of topological transitions from the hyperbolic to elliptical IFCs of twisted $\alpha-\mathrm{MoO}_{3}$ bilayers, occurring for a certain 'magic' twist angle of the layers [36, 37]. Despite the impressive progress in the field of in-plane hyperbolic phonon polaritons of vdW materials, the natural hyperbolic surface plasmon polaritons (SPPs) have not been observed until very recently [38].

Semimetal $\mathrm{WTe}_{2}$ is a layered material with a pronounced anisotropic in-plane electromagnetic response [39]. Thin films made of $\mathrm{WTe}_{2}$ host a wide range of remarkable electronic properties, such as extremely high mobility [40] and tunability of the carrier density by electrostatic gating [41, 42] and Mo doping [43]. Reflection measurements on bulk $\mathrm{WTe}_{2}$ crystals revealed temperature-dependent anisotropic bulk plasma frequencies with optical scattering rates as low as $0.25 \mathrm{~cm}^{-1}$ at $6 \mathrm{~K}[39,44]$. Moreover, the in-plane anisotropy of $\mathrm{WTe}_{2}$ was also investigated by angle-dependent and polarized Raman spectroscopy [45]. Most recently, far-infrared absorption spectroscopy has provided the first experimental evidence that thin films of $\mathrm{WTe}_{2}$ can support SPPs with the in-plane hyperbolic topology in the wavelength range from 16 to $23 \mu \mathrm{m}$ [38]. This study demonstrated topological transitions from the elliptic to hyperbolic regime by mapping the IFCs of plasmons and revealed the strong temperature dependencies of the SPPs frequency and anisotropy. However, there is a lack of research in the literature on the investigation of how the hybridization of SPPs of an anisotropic vdW material (AvdWM), e.g. WTe ${ }_{2}$, and isotropic SPPs of graphene can improve functionality of the leaded heterostructure.

In this paper, we first obtain the dispersion relation and transmission coefficient of hybrid anisotropic-isotropic SPPs supported by a parallel-plate waveguide composed of an AvdWM and graphene. Taking $\mathrm{WTe}_{2}$ parameters in the calculations - as an experimentally realized vdW material with in-plane hyperbolic responses - the impact of the losses on the modal characteristics of SPPs supported by the AvdWM is firstly examined via pure analytical and numerical calculations. We then investigate how the coupling of the anisotropic layer to a graphene sheet in a parallel-plate waveguide heterostructure leads to a control on the in-plane propagation and dispersion topology of the hybrid SPPs by changing the spacer thickness and the graphene chemical potential. Moreover, it is found that due to different coupling regimes, hybridization of anisotropic-isotropic SPPs can enhance the propagation length (PL) and spatial localization of the guided modes. Consequently, the functionalities of the vdW heterostructure for the in-plane and out-of-plane infrared nanophotonics can be improved. 


\section{Theory}

One of the primary goals of this study is to investigate how the presence of losses affects the modal features of the AvdWM (figure 1(a)) analyzed in [38]. We also aim to explore if the presence of a graphene layer in a AvdWM/spacer/G/sub parallel-plate waveguide structure, where ' $G$ ' and 'sub' stand for graphene and substrate, can enhance these features. It is assumed that the AvdWM is separated from the graphene sheet located on a substrate by a dielectric spacer of thickness $l$ (figure 1(b)). Following the previous studies [46, 47], we begin our analysis by deriving an exact dispersion relation of the hybrid guided modes supported by the considered parallelplate waveguide structure. This relation will then be used to plot the dispersion curves and calculate the IFCs of the modes, which will provide valuable information for interpreting the results of the numerical simulations. Note that the considered structure can also be investigated by alternative approaches like coupled mode theory $[48,49]$.

In what follows, we take the two components of the anisotropic optical conductivity tensor of AvdWM, $\underline{\sigma}=$ $\operatorname{diag}\left(\sigma_{x x}, \sigma_{y y}, 0\right)$, to be given by $[19,38]$

$$
\sigma_{j j}(\omega, T)=\frac{\mathrm{i}}{\pi} \frac{D_{j j}}{(\omega+\mathrm{i} \Gamma)}+\frac{\mathrm{i}}{\pi} \frac{\omega S_{j j}}{\left(\omega^{2}-\omega_{\mathrm{b}}^{2}+\mathrm{i} \omega \eta\right)}
$$

where $j=x, y, D_{j j}$ and $S_{j j}$ are the spectral weights, $\omega_{\mathrm{b}}$ is the interband resonance frequency, and $\Gamma$ and $\eta$ are the scattering widths of the intraband and interband transitions, respectively. The parameter values here are borrowed from [38] at $T=10 \mathrm{~K}$. The real and imaginary parts of $\sigma_{x x}$ and $\sigma_{y y}$ are plotted in figure 1(c). The hyperbolic dispersion region that is highlighted in gray is seen to span from $\omega=426.7 \mathrm{~cm}^{-1}$ to $\omega=622.9 \mathrm{~cm}^{-1}$.

The frequency and temperature dependencies of the optical conductivity of the graphene sheet, $\sigma_{\mathrm{g}}=\sigma_{\mathrm{g}}^{\text {intra }}+\sigma_{\mathrm{g}}^{\text {inter }}$, are modeled by the expressions [50]

$$
\begin{aligned}
\sigma_{\mathrm{g}}^{\text {intra }}(\omega, T)=\frac{e^{2}}{4 \hbar} & \frac{\mathrm{i}}{2 \pi} \frac{16 k_{\mathrm{B}} T}{\hbar \Omega} \ln \left[2 \cosh \left(\frac{\mu}{2 k_{\mathrm{B}} T}\right)\right], \\
\sigma_{\mathrm{g}}^{\text {inter }}(\omega, T)=\frac{e^{2}}{4 \hbar} & {\left[\frac{1}{2}+\frac{1}{\pi} \arctan \left(\frac{\hbar \Omega-2 \mu}{2 k_{\mathrm{B}} T}\right)\right.} \\
& \left.-\frac{\mathrm{i}}{2 \pi} \ln \frac{(\hbar \Omega+2 \mu)^{2}}{(\hbar \Omega-2 \mu)^{2}+\left(2 k_{\mathrm{B}} T\right)^{2}}\right],
\end{aligned}
$$

where $-e$ is the electron charge, $\hbar$ is the reduced Plank constant, $k_{\mathrm{B}}$ is the Boltzmann constant, $\Omega=\omega+\mathrm{i} \tau^{-1}, \tau$ is the electron relaxation time, assumed to be $0.3 \mathrm{ps}$, and $\mu$ is the chemical potential.

To obtain the dispersion relation of the supported guided modes in a rather general case, we consider the spacer to be a uniaxial anisotropic medium of permittivity $\underline{\varepsilon}=$ $\operatorname{diag}\left(\varepsilon_{t}, \varepsilon_{t}, \varepsilon_{z}\right)$. In this case, the electric field inside the spacer is a linear combination of ordinary (o) and extraordinary (e) waves, and the electric field in the entire structure is given by [51]

$$
\begin{aligned}
& \boldsymbol{E}(x, y, z, t) \\
& =\mathrm{e}^{\mathrm{i}\left(\beta_{x} x+\beta_{y} y-\omega t\right)} \\
& \quad \times \begin{cases}\boldsymbol{E}_{-} \mathrm{e}^{q_{\mathrm{s}}(z+l / 2)}, \\
\boldsymbol{E}_{+}^{\mathrm{o}} \mathrm{e}^{q_{0} z}+\boldsymbol{E}_{-}^{\mathrm{o}} \mathrm{e}^{-q_{0} z}+\boldsymbol{E}_{+}^{\mathrm{e}} \mathrm{e}^{q_{\mathrm{e}} z}+\boldsymbol{E}_{-}^{\mathrm{e}} \mathrm{e}^{-q_{\mathrm{e}} z}, & |z|<l / 2 \\
E_{+} \mathrm{e}^{-q_{\mathrm{a}}(z-l / 2)}, & z>l / 2\end{cases}
\end{aligned}
$$

where

$$
\begin{gathered}
\boldsymbol{E}_{ \pm}=\left(E_{ \pm x}, E_{ \pm y}, \pm \frac{\mathrm{i}}{q_{\mathrm{a}, \mathrm{s}}}\left(\beta_{x} E_{ \pm x}+\beta_{y} E_{ \pm y}\right)\right), \\
\boldsymbol{E}_{ \pm}^{\mathrm{o}}=E_{ \pm}^{\mathrm{o}}\left(\beta_{y},-\beta_{x}, 0\right), \\
\boldsymbol{E}_{ \pm}^{\mathrm{e}}=E_{ \pm}^{\mathrm{e}}\left(\mp \frac{\mathrm{i} \beta_{x} q_{\mathrm{e}}}{\varepsilon_{t} \beta_{0}}, \mp \frac{\mathrm{i} \beta_{y} q_{\mathrm{e}}}{\varepsilon_{t} \beta_{0}},-\beta_{0}-\frac{q_{\mathrm{e}}^{2}}{\varepsilon_{t} \beta_{0}}\right),
\end{gathered}
$$

and where $q_{\mathrm{a}}=\sqrt{\beta^{2}-\varepsilon_{\mathrm{a}} \beta_{0}^{2}}, \quad q_{\mathrm{s}}=\sqrt{\beta^{2}-\varepsilon_{\mathrm{s}} \beta_{0}^{2}}, \quad q_{\mathrm{o}}=$ $\sqrt{\beta^{2}-\varepsilon_{t} \beta_{0}^{2}}, q_{\mathrm{e}}=\sqrt{\left(\varepsilon_{t} / \varepsilon_{z}\right)\left(k^{2}-\varepsilon_{z} \beta_{0}^{2}\right)}, \beta^{2}=\beta_{x}^{2}+\beta_{y}^{2}$, and $\beta_{0}=\omega / c$. By applying the boundary conditions at $z= \pm l / 2$ [52] and after some algebra, we arrive at the dispersion relation of the guided hybrid plasmonic modes supported by the AvdWM/spacer/G/sub structure in the form

$$
\tanh \left(q_{\mathrm{e}} l\right)=-A / B,
$$

where coefficients $A$ and $B$ are given in the supporting information (available online at stacks.iop.org/JPD/54/ 455102/mmedia).

As a complementary approach to analyze our structure, we use the transfer matrix method to calculate its transmissivity for different wavenumbers of the incident light. Considering the incident, reflected, and transmitted electric fields as $\boldsymbol{E}_{+, i}$, $\boldsymbol{E}_{+, r}$, and $\boldsymbol{E}_{-, t}$ in the calculations we arrive at the following matrix equation

$$
\left(\begin{array}{l}
E_{+x, i} \\
E_{+y, i} \\
E_{+x, r} \\
E_{+x, r}
\end{array}\right)=M\left(\begin{array}{c}
E_{-x, t} \\
E_{-y, t}
\end{array}\right) .
$$

Finally, the transmission coefficient of the AvdWM/spacer/ $\mathrm{G} /$ sub structure can be obtained as (for the details of the derivations and definition of the $M$ matrix please see the supporting information file)

$$
T=\frac{\left|E_{-x, t}\right|^{2}+\left|E_{-y, t}\right|^{2}+\left|\beta_{x} E_{-x, t}+\beta_{y} E_{-y, t}\right|^{2} / q_{\mathrm{s}}^{2}}{\left|E_{+x, i}\right|^{2}+\left|E_{+y, i}\right|^{2}+\left|\beta_{x} E_{+x, i}+\beta_{y} E_{+y, i}\right|^{2} / q_{\mathrm{a}}^{2}} .
$$

It can be readily verified that, taking $\sigma_{\mathrm{g}} \rightarrow 0$ in equations (5) and (7) and the corresponding relations mentioned in the supporting information file, we arrive at our previous results for the guided modes of an AvdWM placed on a uniaxial anisotropic thin film of hBN [11]. 
(a)

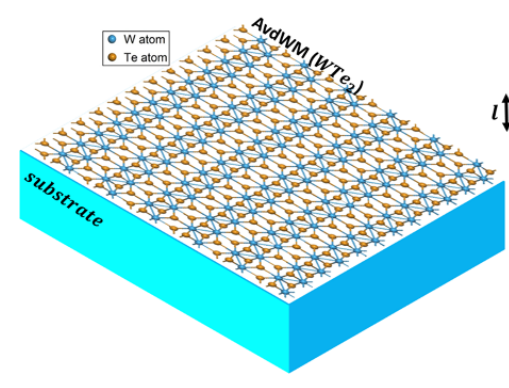

(b)

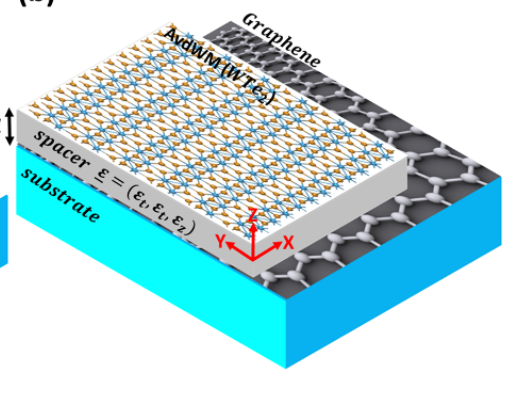

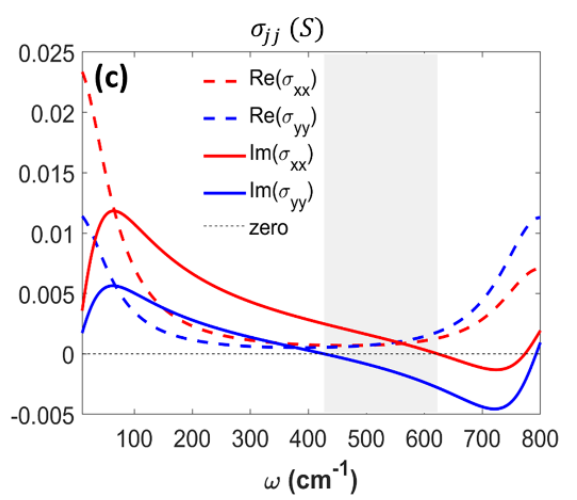

Figure 1. Schematics of (a) AvdWM/sub and (b) AvdWM/spacer/G/sub structures and (c) components of WTe 2 conductivity (as an experimentally realized AvdWM) along the $x$ and $y$ axes; the conductivity dispersion agrees with the data of [38]. Shaded in gray is the region of hyperbolic dispersion.

\section{Results and discussion}

In section 3.1, we analyze the modal characteristics of the anisotropic SPPs supported by a AvdWM/sub structure at $\omega=424 \mathrm{~cm}^{-1}$ and $\omega=562 \mathrm{~cm}^{-1}$ in the presence of losses in AvdWM. The two frequencies of interest correspond to the $\sigma$ near-zero and hyperbolic topologies, respectively. The modal features are examined for the low-loss (or, strictly speaking, lossless) and lossy cases. For the mode profiles that are obtained using finite difference time domain (FDTD) calculations [53], for numerical convergence, the low-loss scenario corresponds to $\sigma_{j j}=0.1 \sigma^{\prime}{ }_{j j}+i \sigma^{\prime \prime}{ }_{j j}$ (that can well present single or multilayers of $\mathrm{WTe}_{2}$ ). For the lossy scenario, the conductivity $\left(\sigma_{j j}=\sigma^{\prime}{ }_{j j}+i \sigma^{\prime \prime}{ }_{j j}\right)$ is taken from [38] and shown in figure 1(c). However, in the analytical calculations related to the IFCs, $\sigma^{\prime}{ }_{j j}=0$ for the lossless scenario. In section 3.2, the effect of the graphene layer, leading to the coupling of the graphene SPPs to the AvdWM SPPs, is investigated for two frequencies in the low-loss and lossy cases. Finally, the dispersion curves, PL, and localization length (LL) of the hybrid modes are analyzed for a wide range of frequencies. Note that, the low-loss and lossy scenarios for graphene and $\mathrm{WTe}_{2}$ in section 3.2 are similar to the ones applied for $\mathrm{WTe}_{2}$ in section 3.1; i.e. regarding the low-loss scenario, for the FDTD calculations related to the mode profiles, $\sigma_{j j, \mathrm{~g}}=0.1 \sigma^{\prime}{ }_{j j, \mathrm{~g}}+$ $i \sigma^{\prime \prime}{ }_{j j, \mathrm{~g}}$ and for the analytical calculations related to the IFCs and figure $7, \sigma_{j j, \mathrm{~g}}^{\prime}=0$. Moreover, for the FDTD calculations related to all top- and side-view mode profiles, a $z$-polarized electric dipole is located at $x=y=0,10 \mathrm{~nm}$ away from the AvdWM layer, and the top-view mode profiles are calculated in a plane which is $5 \mathrm{~nm}$ away from the surfaces of the structures. Note that both graphene and $\mathrm{WTe}_{2}$ have been modeled using their corresponding surface conductivity in the FDTD calculations. Moreover, for our results in this study, the spacer layer and the substrate are considered to be an isotropic medium with refractive index of $1.5(\mathrm{KCl}$ can be mentioned as the material example in this regard). It is worth mentioning that $\mathrm{hBN}$ can be another practical material for the spacer layer. Further investigations (results not illustrated) show that by considering $\mathrm{hBN}$ as the spacer layer $\left(\varepsilon_{t}=4.27\right.$ and $\varepsilon_{z}=6$ for the range of frequency in our study), the reported results in
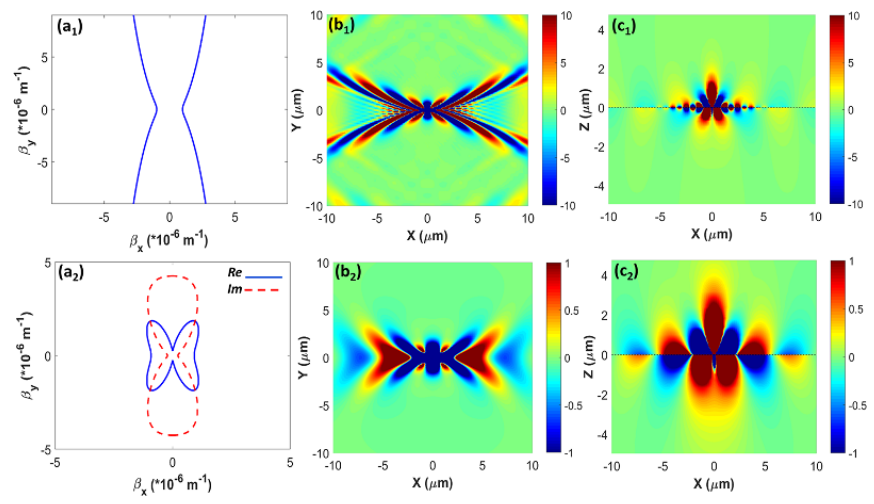

Figure 2. Modal characteristics of $\sigma$-near-zero SPPs supported by $\left(a_{1}\right)-\left(c_{1}\right)$ low-loss and $\left(a_{2}\right)-\left(c_{2}\right)$ lossy AvdWM/sub structures at $\omega=424 \mathrm{~cm}^{-1}$; panels $\left(\mathrm{a}_{\mathrm{i}}\right),\left(\mathrm{b}_{\mathrm{i}}\right)$, and $\left(\mathrm{c}_{\mathrm{i}}\right)$ represent the IFCs, top views, and side views of $E_{z}$, respectively. The real and imaginary parts in $\left(\mathrm{a}_{2}\right)$ are shown with solid blue and dashed red curves, respectively. The dashed horizontal lines in $\left(\mathrm{c}_{\mathrm{i}}\right)$ show the position of the $\mathrm{WTe}_{2}$ sheet.

section 3.2 and the main concluded points still remain valid. Moreover, it should be noted that the IFCs in figures 2-5 and figure 8 are obtained based on equation (5) and panels (a)-(d) of figure 7 are plotted using equation (7).

\subsection{Modal characteristics of SPPS of AvdWM/sub structure}

In the previous study [38], the ohmic losses of AvdWM were neglected and their effect on mode propagation remained unexamined. Therefore, we first investigate the effect of losses on the IFCs of SPPs supported by the AvdWM/sub structure at $\omega=424 \mathrm{~cm}^{-1}$ and $\omega=562 \mathrm{~cm}^{-1}$.

The IFC of the SPPs supported by the lossless AvdWM/sub structure at $\omega=424 \mathrm{~cm}^{-1}$ is shown in figure $2\left(a_{1}\right)$ and agrees well with the results of [38]. The imaginary part of the IFC in this case is negligible and, therefore, not shown in the figure. Because $\sigma^{\prime \prime}{ }_{y y} \sim 0$ (thus $\sigma^{\prime \prime}{ }_{x x} \gg \sigma^{\prime \prime}{ }_{y y}$ ) at the considered frequency (see figure 1(c)), the IFC has an anisotropic elliptic or $\sigma$-near-zero topology that favors mode propagation in the $x$ direction. Figures $2\left(b_{1}\right)$ and $\left(c_{1}\right)$ show the top and side views 


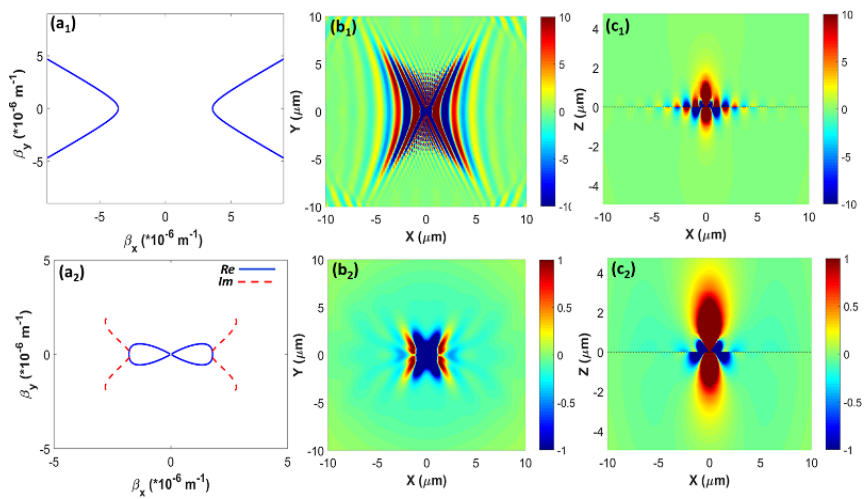

Figure 3. The same as in figure 2 but for $\omega=562 \mathrm{~cm}^{-1}$.

of $E_{z}$. In agreement with the IFC plot, figure $2\left(\mathrm{~b}_{1}\right)$ shows mode canalization in the $x$ direction, which is confirmed by the value of $\tan ^{-1}\left( \pm \sqrt{\left|\frac{\sigma^{\prime \prime}{ }_{y y}}{\sigma^{\prime \prime} x_{x x}}\right|}\right)$ or $\tan ^{-1}\left(\left|\frac{q_{x}^{\prime}}{q_{y}^{\prime}}\right|\right)[8,10]$.

The IFC corresponding to realistic ohmic losses differs considerably from the low-loss scenario. The dashed red curve in figure 2( $\left.a_{2}\right)$ shows that the supported anisotropic SPP in this case is characterized by large $\beta^{\prime \prime}{ }_{x, y}$. The propagation of SPP is, therefore, more canalized in the $x$ direction, yet attenuates faster than in the low-loss case (cf figures $2\left(b_{1}\right)$ and $\left(b_{2}\right)$ ). The side view of $E_{z}$ indicates that the localization of SPPs to the surface of AvdWM strengthens with losses (cf figures 2(c $\left.c_{1}\right)$ and $\left(\mathrm{c}_{2}\right)$ considering the scale difference).

According to figure $1(\mathrm{c}), \quad\left|{\sigma^{\prime \prime}}_{y y} / \sigma^{\prime \prime}{ }_{x x}\right| \approx 2$ and $\sigma^{\prime \prime}{ }_{x x} \sigma^{\prime \prime}{ }_{y y}<0$ at $\omega=562 \mathrm{~cm}^{-1}$. Therefore, the IFC and the top-view of $E_{z}$ of the low-loss SPPs have hyperbolic shapes (figures $3\left(a_{1}\right)$ and $\left(b_{1}\right)$ ). In the presence of losses, the structure supports SPP with large values of $\beta^{\prime \prime}{ }_{x, y}$ and the respective IFC is noticeably different (figure $3\left(a_{2}\right)$ ). The comparison of figures $3\left(b_{1}\right)$ and $\left(b_{2}\right)$ shows that the losses considerably reduce the in-plane PL of the SPPs. Therefore, $\mathrm{WTe}_{2}$ can find potential application in light absorbers based on AvdWMs [54]. Like figure 2 , the side-view mode profiles in figures $3\left(c_{1}\right)$ and $\left(c_{2}\right)$ show that the supported SPP is strongly localized for both low-loss and lossy cases.

The analysis of modal characteristics of the AvdW$\mathrm{M} / \mathrm{sub}$ SPP at another frequency in the hyperbolic region, $\omega=424 \mathrm{~cm}^{-1}$, is presented in supplementary information. According to figure $\mathrm{S} 2$, while the hyperbolic response at this frequency is stronger than the response observed at $562 \mathrm{~cm}^{-1}$, the SPP modal characteristic still considerably modified by the presence of losses.

\subsection{Hybrid guided modes of AvdWM/spacer/G/sub heterostructure}

As we have seen in the previous section, the modal features of SPPs supported by the AvdWM/sub structure are strongly affected by the ohmic losses in the thin films of lossy AvdWMs. We will now investigate how the presence of graphene in the AvdWM/spacer/G/sub parallel = plate waveguide structure (figure 1(b)) can enhance the modal characteristics of the
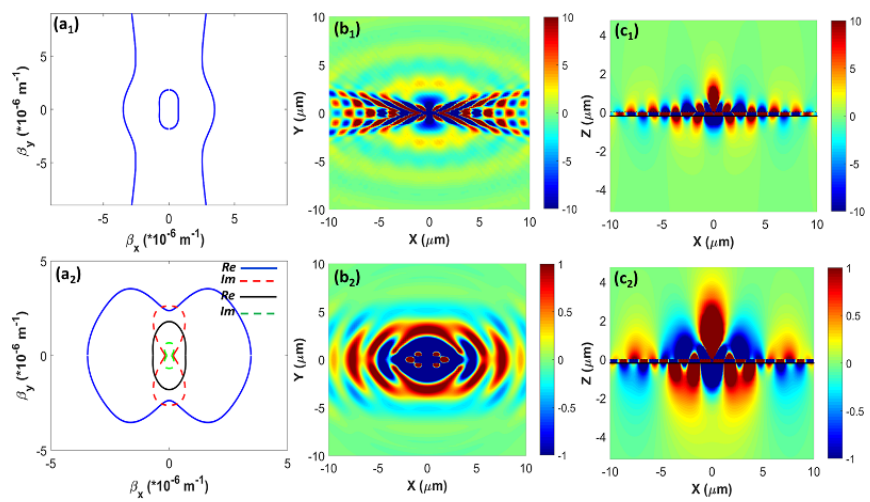

Figure 4. Modal characteristics of $\sigma$-near-zero SPPs supported by $\left(a_{1}\right)-\left(c_{1}\right)$ low-loss and $\left(a_{2}\right)-\left(c_{2}\right)$ lossy AvdWM/spacer/G/sub structures at $\omega=424 \mathrm{~cm}^{-1}$. Panels $\left(a_{i}\right),\left(b_{i}\right)$, and $\left(c_{i}\right)$ show IFCs, top views, and side views of $E_{z}$, respectively. The solid blue (solid black) and dashed red (dashed green) curves in $\left(\mathrm{a}_{2}\right)$ are the real and the corresponding imaginary parts of the outer (inner) IFCs. The dashed and solid horizontal lines in $\left(c_{1}\right)$ and $\left(c_{2}\right)$ show the AvdWM film and graphene, respectively.

supported hybrid SPPs. The graphene sheet and the spacer layer in the structure give us $\mu$ and $l$ as two degrees of freedom to control the modal features of the supported anisotropicisotropic hybrid SPPs. Unless stated otherwise, it is assumed that $\mu=1 \mathrm{eV}$ and $l=200 \mathrm{~nm}$. The analysis of a similar structure with $l=20 \mathrm{~nm}$ can be found in supporting information.

Figure $4\left(a_{1}\right)$ shows the IFC of the hybrid SPP supported by the AvdWM/spacer/G/sub structure in the low-loss scenario at $\omega=424 \mathrm{~cm}^{-1}$. Its comparison with figure $2\left(a_{1}\right)$ shows that the hybridization of the AvdWM SPP with the graphene SPP yields an additional branch of the IFC. The outer branch is seen to be flatter with respect to the $\beta_{y}$ axis, as compared to the ICF of the AvdWM/sub SPPs in figure 2( $\left.a_{1}\right)$. This makes the SPP of the AvdWM/spacer/G/sub structure more canalized toward the $x$ direction than in the case when the graphene sheet is absent. This point is clearly observed once figures $2\left(b_{1}\right)$ and $4\left(b_{1}\right)$ are compared. Therefore, the presence of graphene affects the inplane pattern of SPP propagation.

The solid-blue contour in figure $4\left(a_{2}\right)$ shows that the ohmic losses considerably modify the outer branch of the IFC while also resulting in large values of $\beta^{\prime \prime}{ }_{x, y}$ shown by the dashed red curve. The shape of the dashed green curve, corresponding to the solid black inner contour, indicates that this contour is less affected by losses than the outer one. The comparison of figures $2\left(b_{2}\right)$ and $4\left(b_{2}\right)$ shows that the SPP in the AvdW$\mathrm{M} /$ spacer/G/sub structure propagates further and with stronger canalization than in the AvdWM/sub structure. Therefore, the presence of graphene partially mitigates the effect of ohmic losses.

The modal features of the SPP supported by the AvdW$\mathrm{M} /$ spacer/G/sub structure at $\omega=562 \mathrm{~cm}^{-1}$ are presented in figure 5. As we know, in this case the AvdWM/sub structure supports an SPP with hyperbolic dispersion. Consequently, the outer branch of the IFC in the figure is a somewhat modified hyperbolic dispersion branch while the inner branch is fully due to the plasmon-plasmon hybridization. This conclusion 

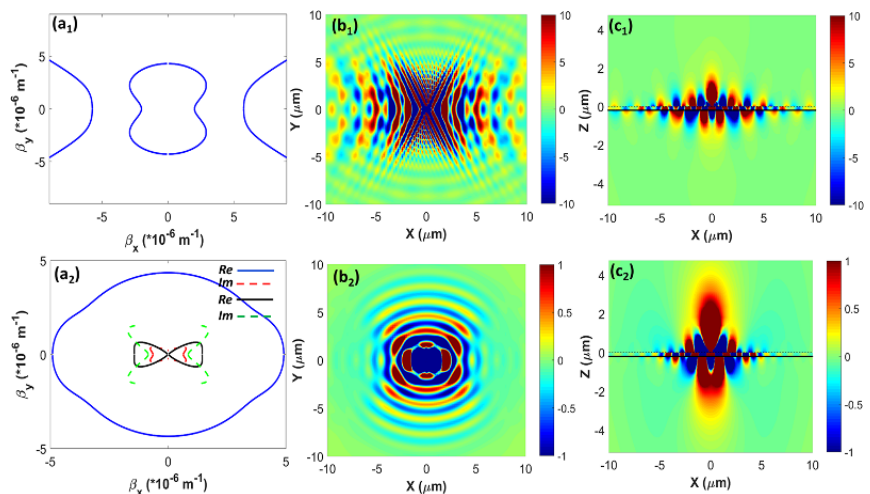

Figure 5. The same as in figure 4 but for $\omega=562 \mathrm{~cm}^{-1}$.

is confirmed by the comparison of figures $3\left(a_{1}\right)$ and $5\left(a_{1}\right)$. The fact that the inner branch is mostly oriented along the $\beta_{y}$ axis makes the SPP in figure $5\left(b_{1}\right)$ feature both the directional propagation of the hyperbolic SPPs in figure $3\left(b_{1}\right)$ and the strong canalization in the $x$ direction. The same conclusion can be drawn for $\omega=476 \mathrm{~cm}^{-1}$ by comparing the mode profiles in figures $\mathrm{S} 2\left(\mathrm{a}_{1}\right),\left(\mathrm{b}_{1}\right)$ and $\mathrm{S} 3\left(\mathrm{a}_{1}\right),\left(\mathrm{b}_{1}\right)$ in supporting information.

Figure $5\left(a_{2}\right)$ illustrates the impact of losses on the branches of the IFC at $\omega=562 \mathrm{~cm}^{-1}$. The blue (black) curve shows $\beta^{\prime}{ }_{x, y}$ and the red (green) curve shows the corresponding $\beta^{\prime \prime}{ }_{x, y}$ of the outer (inner) IFC branch. Because the outer branch corresponding to small $\beta^{\prime \prime}{ }_{x, y}$ is mostly inclined along the $\beta_{x}$ axis, the hybrid SPP propagates predominantly in the $y$ direction (figure $5\left(b_{2}\right)$ ). The comparison of figures $3\left(b_{2}\right)$ and $5\left(b_{2}\right)$ shows that the presence of graphene noticeably increases the PL of the guided mode and significantly changes its propagation direction.

The side-views of the mode profiles in figures $4\left(c_{i}\right)$ and $5\left(c_{i}\right)$ show the extent of localization of the guided modes inside the spacer and their characteristic decay lengths in the air and substrate. It should be noted that by analyzing the side-view $Y Z$ mode profiles it is understood that the supported guided modes are also highly localized in this plane. As we will see in figure 8(c), this localization is stronger than in the case of AvdWM SPPs.

The top-view mode profiles shown in figure 6 for the lowloss and lossy structures at $562 \mathrm{~cm}^{-1}$ show how the chemical potential of graphene affects the propagation direction of the guided modes. The resemblance of figures $3\left(b_{i}\right)-6\left(a_{i}\right)$ indicates that the features of the hybrid SPPs of the AvdWM/spacer/G/sub structure with low $\mu$ are similar to those of the SPPs guided by the AvdWM/sub structure. The increase of $\mu$ modifies the propagation direction and increases the PL of the SPPs (figures $6\left(b_{i}\right)$ and $\left(c_{i}\right)$ ).

So far, we have only investigated the modal characteristics at single frequencies. Hereinafter, we analyze the dispersion of the hybrid SPPs in the presence (figure 7) and absence (figure 8) of losses.

Let us first neglect the ohmic losses and use equation (7) to visualize the dispersion of SPPs supported by the Avd$\mathrm{WM} / \mathrm{sub}$ structure and the AvdWM/spacer/G/sub structure
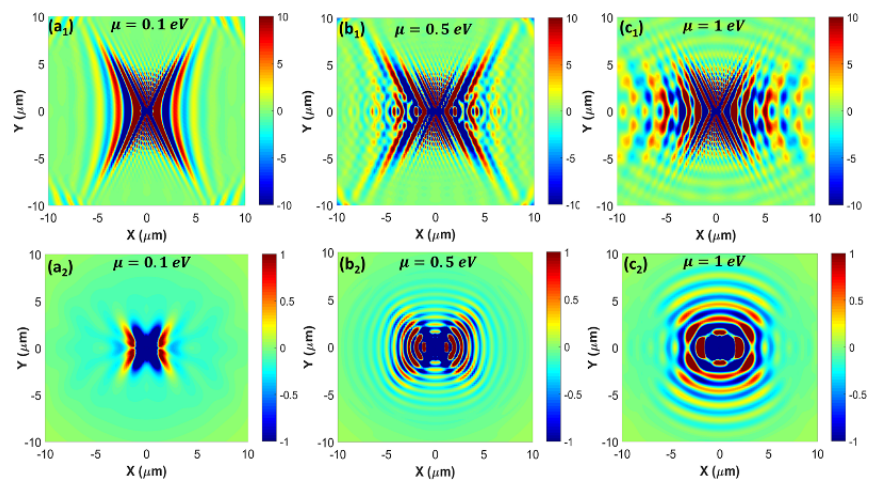

Figure 6. Top-view of $E_{z}$-field profiles of hybrid SPPs supported by $\left(a_{1}\right)-\left(c_{1}\right)$ low-loss and $\left(a_{2}\right)-\left(c_{2}\right)$ lossy AvdWM/spacer/G/sub structures for $\omega=562 \mathrm{~cm}^{-1}$ and different values of the chemical potential of graphene.
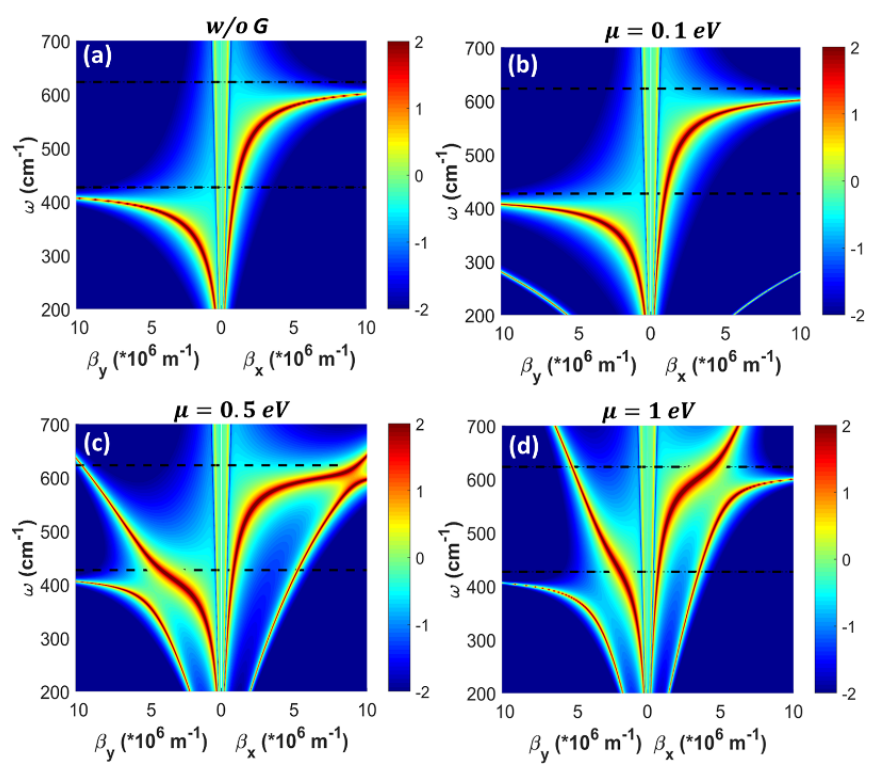

Figure 7. SPP dispersion in lossless (a) AvdWM/sub structure and (b)-(d) AvdWM/spacer/G/sub structure with $t=200 \mathrm{~nm}$ for different values of chemical potential of graphene. Dashed horizontal lines bound the hyperbolic dispersion region of the AvdWM, where $\sigma^{\prime \prime}{ }_{x x} \sigma^{\prime \prime}{ }_{y y}<0$.

with different values of $\mu$. The respective density plots are presented in figure 7. In agreement with the results of [38], the AvdWM/sub structure supports the propagation of hyperbolic SPPs only along the $x$ direction (figure 7(a)). The calculations show that the AvdWM/spacer/G/sub structure does not support hybrid SPPs when $\mu=0.05 \mathrm{eV}$ (the density plot is not shown). The increase of $\mu$ to $0.1 \mathrm{eV}$ yields two additional SPP branches for $\omega<300 \mathrm{~cm}^{-1}$ without affecting the dispersion of the AvdWM SPPs (figure 7(b)). This is a weak hybridization regime, which occurs for chemical potentials below $0.4 \mathrm{eV}$ and is characterized by a simple blueshift of graphene SPP branches with $\mu$. The strong hybridization regime is realized for $\mu>0.45 \mathrm{eV}$ when the coupling between the isotropic graphene SPPs and the anisotropic AvdWM SPPs noticeably transforms the dispersion of the hybrid guided modes. The 

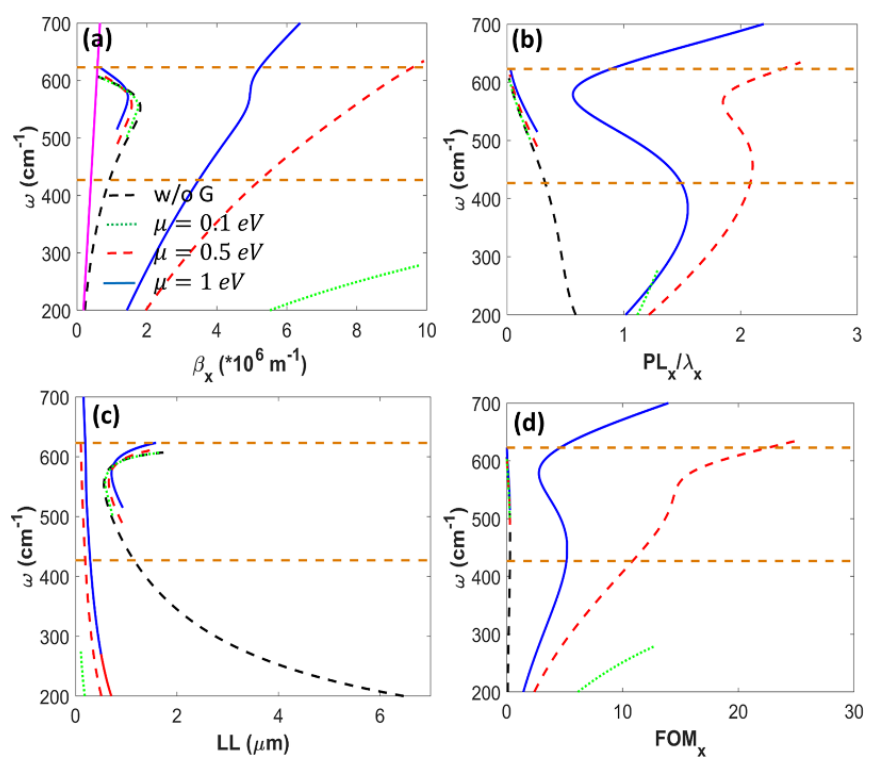

Figure 8. Modal characteristics of SPPs of AvdWM/sub structure (dashed black curves) and AvdWM/spacer/G/sub structure (solid blue, dashed red, and dotted green curves) with $l=200 \mathrm{~nm}$ in the presence of losses: (a) frequency dispersion, (b) $\mathrm{PL}_{x} / \lambda_{x}$, (c) LL in micron, and (d) $\mathrm{FOM}_{x}$. Dashed horizontal lines are the boundaries of the hyperbolic dispersion region of the AvdWM. The solid pink curve in (a) represents the light-line of the substrate.

strong hybridization regime is characterized by two distinct anticrossings in the SPP dispersion, which are clearly visible for $\mu=0.5 \mathrm{eV}$ in figure 7(c) and become less pronounced for $\mu=1 \mathrm{eV}$ in figure $7(\mathrm{~d})$. A similar analysis of the dispersion characteristics of the lossless hybrid SPPs for $l=20 \mathrm{~nm}$ is given in supporting information (figure S7).

As we have seen, ohmic losses is an impediment to anisotropic guiding characteristic of the considered AvdWM. It was found that introducing graphene into the AvdWM/spacer/G/sub structure can significantly increase the PL and reduce the LL of the guided modes. We now-taking real values of $\omega$ and complex values of $\beta$ in the calculations based on equation (5) - analyze the dispersion of these two lengths and the figure-of-merit (FOM) in the $x$ direction for the AvdW$\mathrm{M} / \mathrm{sub}$ and AvdWM/spacer/G/sub structures using the following definitions [55]:

$$
\begin{gathered}
\mathrm{PL}_{x}=1 /\left(2 \beta^{\prime \prime}{ }_{x}\right), \\
\lambda_{x}=2 \pi / \beta^{\prime}{ }_{x}, \\
\mathrm{LL}=\frac{1}{2}\left(\frac{1}{\left|\operatorname{Re} q_{\mathrm{a}}\right|}+\frac{1}{\left|\operatorname{Re} q_{\mathrm{s}}\right|}\right), \\
\operatorname{FOM}_{x}=\frac{\mathrm{PL}_{x} / \lambda_{x}}{\operatorname{LL}(\mu \mathrm{m})} .
\end{gathered}
$$

Figure 8(a) shows the dispersion of SPPs supported by the AvdWM/sub and AvdWM/spacer/G/sub structures in the presence of losses. The comparison of the shape of the dashed black curve with figure 7(a) shows that losses dramatically alter the dispersion of the AvdWM/sub SPPs in the hyperbolic region. This conclusion does not hold for low-loss graphene SPPs, as it was demonstrated for thin metallic films [56]. In full agreement with the IFC plots and the results of figure 7 , one can also see that adding a graphene sheet to the structure yields an additional branch in the SPP dispersion. Regardless of $\mu$, the first dispersion branch of the hybrid SPPs with $\beta^{\prime}{ }_{x}<$ $1.5 \times 10^{-6} \mathrm{~m}^{-1}$ resembles the dispersion of the AvdWM/sub SPPs inside the hyperbolic region. The situation is different for the second dispersion branch with $\beta^{\prime}{ }_{x}>1.5 \times 10^{-6} \mathrm{~m}^{-1}$. This branch is like the dispersion in figure 7(b) outside of the hyperbolic region only in the weak hybridization regime when $\mu=0.1 \mathrm{eV}$ (dotted green curve). However, if the hybridization is strong $(\mu=0.5$ or $1 \mathrm{eV})$, the dispersion curves are strongly modified by the losses.

The normalized PL, LL in micron, and FOM of the hybrid SPPs in the AvdWM/sub and AvdWM/spacer/G/sub structures are plotted in figures 8 (b)-(d). The presence of graphene is seen to increase the normalized PL for both dispersion branches. The comparison of the SPP dispersion in the Avd$\mathrm{WM} /$ sub structure (dashed black curve) with the dispersion branches of the hybrid SPPs shows that graphene also reduces the mode localization for the first branch and increases it for the second one. Consequently, $\mathrm{FOM}_{x}$ values of the first branch of the hybrid SPPs of the AvdWM/spacer/G/sub system possess similar values of the AvdWM/sub SPPs, as observed from figure $8(\mathrm{~d})$. On the contrary, the $\mathrm{FOM}_{x}$ values of the second branch of the hybrid modes are considerably larger than the ones of AvdWM/sub SPPs, both inside and outside of the hyperbolic region, especially for the case of $\mu=0.5 \mathrm{eV}$. As a result, in addition to providing two additional degrees of freedom in controlling the anisotropic SPPs of the AvdWM, the presence of graphene leads to considerable enhancement in their in-plane modal features. It is noteworthy that in addition to improving the in-plane characteristic of the supported guided modes, it is also possible to increase out-of-plane functionalities of a metasurface composed of meta-toms of AvdWM, by its integration to a graphene layer as proved in [54]. Further analysis shows that the reduction of the spacer thickness to $l=20 \mathrm{~nm}$ makes the guided anisotropic modes exist at larger $\beta^{\prime}{ }_{x}$ and with greater values of $\mathrm{FOM}_{x}$ as compared to figure 8(a) (see figure S8 in the supplementary information and the discussion that follows). This increases the fabrication complexity by requiring a smaller patterning for mode excitation compared to the case of $l=200 \mathrm{~nm}$. Another point that should be highlighted here is that more investigations proves the conclusions made here for figure 8 are also valid for $T=300 \mathrm{~K}$ (see figure S9 in the supporting information).

\section{Conclusion}

To conclude, via analytical calculations and numerical simulations, we have examined hybrid anisotropic-isotropic SPPs supported by a parallel-plate waveguide composed of an AvdWM and graphene. Taking parameters of $\mathrm{WTe}_{2}$ as an experimentally realized plasmonic material with infrared 
in-plane hyperbolic response, we first investigated IFCs and propagation and localization characteristics of SPPs of the AvdWM. Then, it was examined that how modal characteristics and topology of the anisotropic SPPs can be modified once a graphene sheet was coupled to the AvdWM in a parallel-plate waveguide configuration. We showed that this coupling allows one to tune the modal features and dispersion topology of the supported hybrid SPPs by changing the graphene chemical potential and the spacer layer thickness. It was also shown that owing to the different coupling regime, the propagation and localization characteristics of the hybrid modes are improved compared to the features of the isolated AvdWM SPPs. The proposed strategy can lead to the realization of functional vdW heterostructures for in-plane and out-of-plane infrared nanophotonics.

\section{Data availability statement}

No new data were created or analysed in this study.

\section{Acknowledgments}

E $\mathrm{O}$ and $\mathrm{H} \mathrm{H}$ acknowledge the financial support from TÜBITAK Project under No. 120E422 and partial support from the Turkish Academy of Sciences. I R thanks the Russian Science Foundation (Grant No. 19-13-00332).

\section{Conflict of interest}

The authors declare no conflict of interest.

\section{ORCID iDs}

Hodjat Hajian (D) https://orcid.org/0000-0001-6564-6273

Ivan D Rukhlenko (D) https://orcid.org/0000-0001-5585-4220

George W Hanson (D) https://orcid.org/0000-0001-57928836

Ekmel Ozbay (D) https://orcid.org/0000-0003-2953-1828

\section{References}

[1] Gomez-Diaz J S, Tymchenko M and Alù A 2015 Hyperbolic plasmons and topological transitions over uniaxial metasurfaces Phys. Rev. Lett. 114233901

[2] Kildishev A V, Boltasseva A and Shalaev V M 2013 Planar photonics with metasurfaces Science 3391232009

[3] Liu Y and Zhang X 2013 Metasurfaces for manipulating surface plasmons Appl. Phys. Lett. 103141101

[4] High A A, Devlin R C, Dibos A, Polking M, Wild D S, Perczel J, de Leon N P, Lukin M D and Park H 2015 Visible-frequency hyperbolic metasurface Nature 522 192-6

[5] Yang Y et al 2017 Hyperbolic spoof plasmonic metasurfaces NPG Asia Mater. 9 e428

[6] Nemilentsau A, Low T and Hanson G 2016 Anisotropic 2D materials for tunable hyperbolic plasmonics Phys. Rev. Lett. 116066804

[7] Correas-Serrano D, Gomez-Diaz J S, Alvarez Melcon A and Alù $\mathrm{A} 2016$ Black phosphorus plasmonics: anisotropic elliptical propagation and nonlocality-induced canalization J. Opt. 18104006

[8] Hassani Gangaraj S A, Low T, Nemilentsau A and Hanson G W 2017 Directive surface plasmons on tunable two-dimensional hyperbolic metasurfaces and black phosphorus: Green's function and complex plane analysis IEEE Trans. Antennas Propag. 651174

[9] Nemilentsau A, Stauber T, Gómez-Santos G, Luskin M and Low T 2019 Switchable and unidirectional plasmonic beacons in hyperbolic two-dimensional materials Phys. Rev. B 99201405

[10] Nemilentsau A, Low T and Hanson G 2019 Chiral and hyperbolic plasmons in novel 2D materials Carbon-based Nanoelectromagnetics (Amsterdam: Elsevier) pp 119-38

[11] Hajian H, Rukhlenko I D, Hanson G W, Low T, Butun B and Ozbay E 2020 Tunable plasmon-phonon polaritons in anisotropic 2D materials on hexagonal boron nitride Nanophotonics 93909

[12] Correas-Serrano D, Alù A and Gomez-Diaz J S 2017 Plasmon canalization and tunneling over anisotropic metasurfaces Phys. Rev. B 96075436

[13] Gomez-Diaz J S and Alù A 2016 Flatland optics with hyperbolic metasurfaces ACS Photonics 32211

[14] Cortes C L and Jacob Z 2017 Super-Coulombic atom-atom interactions in hyperbolic media Nat. Commun. 814144

[15] Caldwell J D et al 2014 Sub-diffractional volume-confined polaritons in the natural hyperbolic material hexagonal boron nitride Nat. Commun. 55221

[16] Dai S et al 2014 Tunable phonon polaritons in atomically thin van der waals crystals of boron nitride Science 343 1125-9

[17] Li P, Dolado I, Alfaro-Mozaz F J, Casanova F, Hueso L E, Liu S, Edgar J H, Nikitin A Y, Vélez S and Hillenbrand R 2018 Infrared hyperbolic metasurface based on nanostructured van der Waals materials Science 359 892-6

[18] Li P et al 2020 Collective near-field coupling and nonlocal phenomena in infrared-phononic metasurfaces for nano-light canalization Nat. Commun. 113663

[19] Basov D N, Fogler M M and Garcia de Abajo F J 2016 Polaritons in van der Waals materials Science 354 aag 1992

[20] Low T, Chaves A, Caldwell J D, Kumar A, Fang N X, Avouris P, Heinz T F, Guinea F, Martin-Moreno L and Koppens F 2017 Polaritons in layered two-dimensional materials Nat. Mater. 16 182-94

[21] Low T, Roldán R, Wang H, Xia F, Avouris P, Moreno L M and Guinea F 2014 Plasmons and screening in monolayer and multilayer black phosphorus Phys. Rev. Lett. 113106802

[22] van Veen E, Nemilentsau A, Kumar A, Roldán R, Katsnelson M I, Low T and Yuan S 2019 Tuning two dimensional hyperbolic plasmons in black phosphorus Phys. Rev. Appl. 12014011

[23] Ma W et al 2018 In-plane anisotropic and ultra-low-loss polaritons in a natural van der Waals crystal Nature $562557-62$

[24] Zheng Z et al 2019 A mid-infrared biaxial hyperbolic van der Waals crystal $S c i$. Adv. 5 eaav8690

[25] Hu G, Shen J, Qiu C, Alù A and Dai S 2020 Phonon polaritons and hyperbolic response in van der Waals materials $A d v$. Opt. Mater. 81901393

[26] Wei C, Abedini Dereshgi S, Song X, Murthy A, Dravid V P, Cao T and Aydin K 2020 Polarization reflector/color filter at visible frequencies via anisotropic $\alpha-\mathrm{MoO}_{3} \mathrm{Adv}$. Opt. Mater. 82000088

[27] Abedini Dereshgi S, Folland T G, Murthy A A, Song X, Tanriover I, Dravid V P, Caldwell J D and Aydin K 2020 Lithography-free IR polarization converters via orthogonal in-plane phonons in a-MoO 3 flakes Nat. Commun. 115771

[28] Fei Z et al 2012 Gate-tuning of graphene plasmons revealed by infrared nanoimaging Nature 487 82-85 
[29] Chen J et al 2012 Optical nano-imaging of gate-tunable graphene plasmons Nature 487 77-81

[30] Fan Y, Shen N-H, Zhang F, Zhao Q, Wu H, Fu Q, Wei Z, Li H and Soukoulis C M 2019 Graphene plasmonics: a platform for 2D optics Adv. Opt. Mater. 71800537

[31] Chaudhary K, Tamagnone M, Rezaee M, Bediako D K, Ambrosio A, Kim P and Capasso F 2019 Engineering phonon polaritons in van der Waals heterostructures to enhance in-plane optical anisotropy $S c i$. $A d v$. 5 eaau 7171

[32] Gjerding M N, Petersen R, Pedersen T G, Mortensen N A and Thygesen K S 2017 Layered van der Waals crystals with hyperbolic light dispersion Nat. Commun. 8320

[33] Reed B W, Williams D R, Moser B P and Koski K J 2019 Chemically tuning quantized acoustic phonons in $2 \mathrm{D}$ layered $\mathrm{MoO}_{3}$ nanoribbons Nano Lett. 194406

[34] Wu Y et al 2020 Chemical switching of low-loss phonon polaritons in $\alpha-\mathrm{MoO}_{3}$ by hydrogen intercalation Nat. Commun. 112646

[35] Taboada-Gutiérrez J et al 2020 Broad spectral tuning of ultra-low-loss polaritons in a van der Waals crystal by intercalation Nat. Mater. 19964

[36] $\mathrm{Hu} \mathrm{G}$ et al 2020 Topological polaritons and photonic magic angles in twisted $\alpha-\mathrm{MoO}_{3}$ bilayers Nature $\mathbf{5 8 2} 209$

[37] Chen M, Lin X, Dinh T H, Zheng Z, Shen J, Ma Q, Chen H, Jarillo-Herrero P and Dai S 2020 Configurable phonon polaritons in twisted $\alpha-\mathrm{MoO}_{3}$ Nat. Mater. 19 1307-11

[38] Wang C, Huang S, Xing Q, Xie Y, Song C, Wang F and Yan H 2020 Van der Waals thin films of $\mathrm{WTe}_{2}$ for natural hyperbolic plasmonic surfaces Nat. Commun. 111158

[39] Frenzel A J, Homes C C, Gibson Q D, Shao Y M, Post K W, Charnukha A, Cava R J and Basov D N 2017 Anisotropic electrodynamics of type-II Weyl semimetal candidate $\mathrm{WTe}_{2}$ Phys. Rev. B 95245140

[40] Wang L, Gutiérrez-Lezama I, Barreteau C, Ubrig N, Giannini E and Morpurgo A F 2015 Tuning magnetotransport in a compensated semimetal at the atomic scale Nat. Commun. 68892

[41] Fatemi V, Gibson Q D, Watanabe K, Taniguchi T, Cava R J and Jarillo-Herrero P 2017 Magnetoresistance and quantum oscillations of an electrostatically tuned semimetal-to-metal transition in ultrathin $\mathrm{WTe}_{2}$ Phys. Rev. B 95041410
[42] Wang Y et al 2016 Gate-tunable negative longitudinal magnetoresistance in the predicted type-II Weyl semimetal $\mathrm{WTe}_{2}$ Nat. Commun. 713142

[43] Fu D, Pan X, Bai Z, Fei F, Umana-Membreno G A, Song H, Wang X, Wang B and Song F 2018 Tuning the electrical transport of type II Weyl semimetal $\mathrm{WTe}_{2}$ nanodevices by Mo doping Nanotechnology 29135705

[44] Homes C C, Ali M N and Cava R J 2015 Optical properties of the perfectly compensated semimetal $\mathrm{WTe}_{2}$ Phys. Rev. B 92161109

[45] Song Q et al 2016 The in-plane anisotropy of $\mathrm{WTe}_{2}$ investigated by angle-dependent and polarized Raman spectroscopy Sci. Rep. 629254

[46] Hanson G W 2008 Quasi-transverse electromagnetic modes supported by a graphene parallel-plate waveguide J. Appl. Phys. 104084314

[47] Hajian H, Rukhlenko I D, Leung P T, Caglayan H and Ozbay E 2016 Guided plasmon modes of a graphene-coated Kerr slab Plasmonics 11735

[48] Huang W, Liang S, Kyoseva E and Ang L K 2018 Adiabatic control of surface plasmon-polaritons in a 3-layers graphene curved configuration Carbon 127187

[49] Huang W, Liang S, Kyoseva E and Ang L K 2018 A new coupling mechanism between two graphene electron waveguides for ultrafast switching Semicond. Sci. Technol. 33035014

[50] Falkovsky L A 2008 Optical properties of graphene J. Phys.: Conf. Ser. 129012004

[51] Tarkhanian R G 1975 On the theory of surface waves in a uniaxial semiconductor slab Phys. Status Solidi b 72111

[52] Hanson G W 2008 Dyadic Green's functions for an anisotropic, non-local model of biased graphene IEEE Trans. Antennas Propag. 56747

[53] Lumerical Inc. Product Overview (available at: www.lumerical.com/products/ (Accessed 15 August 2021))

[54] Nong J, Wei W, Wang W, Lan G, Shang Z, Yi J and Tang L 2018 Strong coherent coupling between graphene surface plasmons and anisotropic black phosphorus localized surface plasmons Opt. Express 261633

[55] Jablan M, Buljan H and Soljačić M 2009 Plasmonics in graphene at infrared frequencies Phys. Rev. B 80245435

[56] Maier S A 2007 Plasmonics: Fundamentals and Applications (Berlin: Springer) 